\title{
CORRELATIONS BETWEEN AGGREGATE PARTICLE SHAPE PARAMETERS AND SIZE BASED ON IMAGES CAPTURED USING A 3-D LASER SCANNING DEVICE
}

Martin B Mgangira, CSIR Built Environment, P.O Box 395, Pretoria, 0001, South Africa. E-Mail: MMgangira@csir.co.za

Julius Komba, CSIR Built Environment, P.O Box 395, Pretoria, 0001, South Africa. E-Mail: JKomba@csir.co.za

doi: 10.2478/ijpeat-2013-0010

\begin{abstract}
Three dimensional (3-D) laser scanning has recently proved to be an ideal technique for quantifying aggregate particle shape parameters. This is because 3-D laser scanners measure fine details and capture free-form shapes. This paper presents some results of an on-going study, which utilises 3-D laser scanning technology to quantify aggregate shape parameters. Specific correlations and shape parameters have been considered. The results have shown that meaningful correlations exist between 3-D sphericity and aspect ratio as well as between specific surface area and the particle diameter as defined in this paper. The results of the current study are consistent with generally accepted relationships between shape parameters and particle size, further validating the application of the 3-D laser scanning technology.
\end{abstract}

\section{Introduction}

The importance of aggregate shape characteristics in the behaviour of natural and crushed aggregates is well recognized. For this reason, a number of studies have been devoted to the quantification of particle shape in recent years and have shown that shape characteristics influence the bulk properties of the materials (Prowell et. al., 2005; Das, 2006; Santamarina and Cho, 2004; Janoo, 1998). According to Janoo (1998), the development of methods for the description of particle shape can be traced back to the early work by Wadel in 1932 and Krumbein in 1941, who introduced sphericity and roundness parameters. Since then, various techniques have been introduced for the quantification of particle shape, ranging from charts for the visual evaluation of particle shape to those based on the application of image analysis, using 2-D image projections of the particles.

In recent years, the emergence of advanced techniques in image capturing has made it easier to quantify the shape parameters based on complete 3-D images. This requires the use of advanced technologies such as the X-ray computed tomography (X-ray CT) technique and 3-D laser scanning devices. In this paper, particle shape parameters were obtained using the true 3-D images captured by using a laser scanning device. The laser scanning device has been shown to be ideally suitable for this purpose. For example, Anochie-Boateng et. al., 2013 and Anochie-Boaten et. al., 2012 found very good correlations between laser-based aggregate shape indices, such as flakiness index, flat and elongation ratio, and those obtained from the results of the current standard tests used to evaluate aggregate shape properties. Pan and Tutumluer (2010) 
used 3-D laser scanning to validate surface area factors of crushed and uncrushed natural aggregates for asphalt mix designs.

\section{Aggregate Particle Measurement}

\section{3-D Image Acquisition}

In this study a LPX 1200 laser scanning device, manufactured by Roland DGA Corporation was used to scan and capture the aggregate images. The 3-D laser scanner uses a non-contact sensor and a spot-beam triangulation scanning method to scan the particles in three dimensions (Anochie-Boateng et al., 2013). The wavelength of the scanner is about $660 \mathrm{~nm}$, and the highest scanning resolution in this case is 0.1 $\mathrm{mm}(100 \mu \mathrm{m})$. The device operates in both rotation and plane scanning modes to make it suitable to scan different types and sizes of aggregates. In the rotary mode, objects are scanned on a fully integrated rotating table using a laser beam, which travels vertically up the rotating object to generate a digital scan file. In brief, data files are registered and merged into a three-dimensional representation of the aggregate particle. Thus 3-D laser scanning captures the exact size and shape of the aggregate particle. Further details are provided in (Anochie-Boateng et al., 2013).

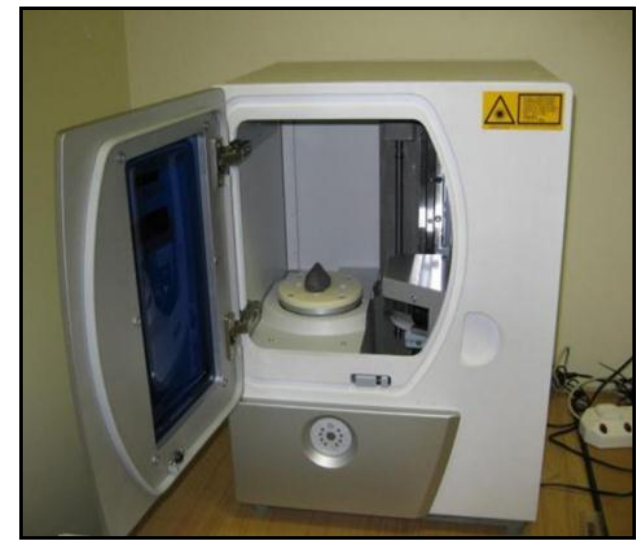

Figure 1. LPX 1200, 3-D Laser scanning device

\section{Data Analysis}

The data files are post-processed using an integrated software package (Rapidform software) which allows automatic calculation of the surface area, volume, and dimensions of individual (details are provided in Anochie-Boateng et. al., (2013). While the object is being scanned, the software captures and streamlines the data editing process to provide high quality scans. The Rapidform software allows users to merge scans for increased quality, change the shape around curved surfaces, sharpen edges, extend shapes, add thickness and perform Boolean operations on polygon surfaces. These features are essential for obtaining accurate morphological properties of aggregates used in road and airport pavements.

In this paper the dimensions of the particle are width, height and depth, defined as follows: (a) the width $\left(\mathrm{d}_{\mathrm{L}}\right)$ is the largest straight-line surface point-to-surface point distance on the particle, (b) the height, the intermediate dimension $\left(\mathrm{d}_{\mathrm{I}}\right)$ is defined similarly, except that it is perpendicular to the length and (c) the depth, the smallest 
dimension $\left(d_{S}\right)$ is perpendicular to both the length and the width. These dimensions are used to define aggregate shape parameters.

The most common particle shape is with respect to elongation ratio and flatness ratio. The flatness ratio is defined as the ratio of the shortest dimension to the intermediate dimension, $\left(\mathrm{d}_{\mathrm{S}} / \mathrm{d}_{\mathrm{I}}\right)$. The elongation ratio is the ratio of the intermediate dimension to the largest dimension $\left(\mathrm{d}_{\mathrm{I}} / \mathrm{d}_{\mathrm{L}}\right)$. Other parameters that are specifically examined are:

- Aspect ratio (AR), defined in this paper as the ratio of the largest dimension to the smallest dimension $\left(\mathrm{d}_{\mathrm{L}} / \mathrm{d}_{\mathrm{S}}\right)$,

- The specific surface area (SSA), defined as the ratio of the mass of the particle to the surface area of the particle,

- Form defined as ratio, $\left(\mathrm{d}_{\mathrm{L}}-\mathrm{d}_{\mathrm{I}}\right) /\left(\mathrm{d}_{\mathrm{L}}-\mathrm{d}_{\mathrm{S}}\right)$,

- Surface area ratio (SAR), defined as the ratio of the particle surface area to the surface area of a sphere of equivalent volume.

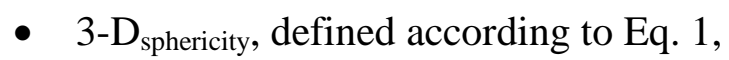

$$
3-D_{\text {sphericity }}=\sqrt[3]{\frac{d_{s} \times d_{1}}{d_{L}^{2}}}
$$

- Parameter SEVA, defined by Eq. 2, which takes into account the particle volume (V) and particle surface area (SA),

$$
S E V A=\frac{\sqrt{36 \pi V^{2}}}{S A}
$$

- The particle diameter, defined by Eq. 3,

$$
d_{P}=\sqrt{d_{I} \times d_{S}}
$$

\section{Result and Discussion}

\section{Particle Shape Classification}

A sample, comprising three particle size classes, was used for the study. The aggregate particles were all obtained from the same parent rock, tillite and subjected to the same crushing method. The evaluation comprised of 30 particles from each of the three particle size classes: (i) material passing the $37.5 \mathrm{~mm}$ sieve but retained on $26.5 \mathrm{~mm}$ sieve, (ii) material passing the $26.5 \mathrm{~mm}$ sieve but retained on $19 \mathrm{~mm}$ sieve and (iii) material passing $19 \mathrm{~mm}$ sieve but retained on $13.2 \mathrm{~mm}$. The three particle size classes give the volume particle sizes; $26.5 \mathrm{~mm}, 19 \mathrm{~mm}$ and $13.2 \mathrm{~mm}$. 
Particle shape can be classified in several ways. Results of the aggregate particle shape, on the basis of flatness ratio and elongation ratio, using the Zingg diagram (Lees, 1964), are presented in Figure 2. The results in Figure 2 were obtained from the spreadsheet analysis, based on the Sneed and Folk triangular diagram (Graham and Midgley, 2000). Observation of the results shows that no specific particle size class falls in a specific shape category. All the particle shape categories are represented in each particle size class. The results of the classification using the triangular diagram are shown in Figure 3 and reveal that particles are mostly compact in shape. The results are consistent with those in Figure 2, corresponding to the Disc-Spheriod shape.

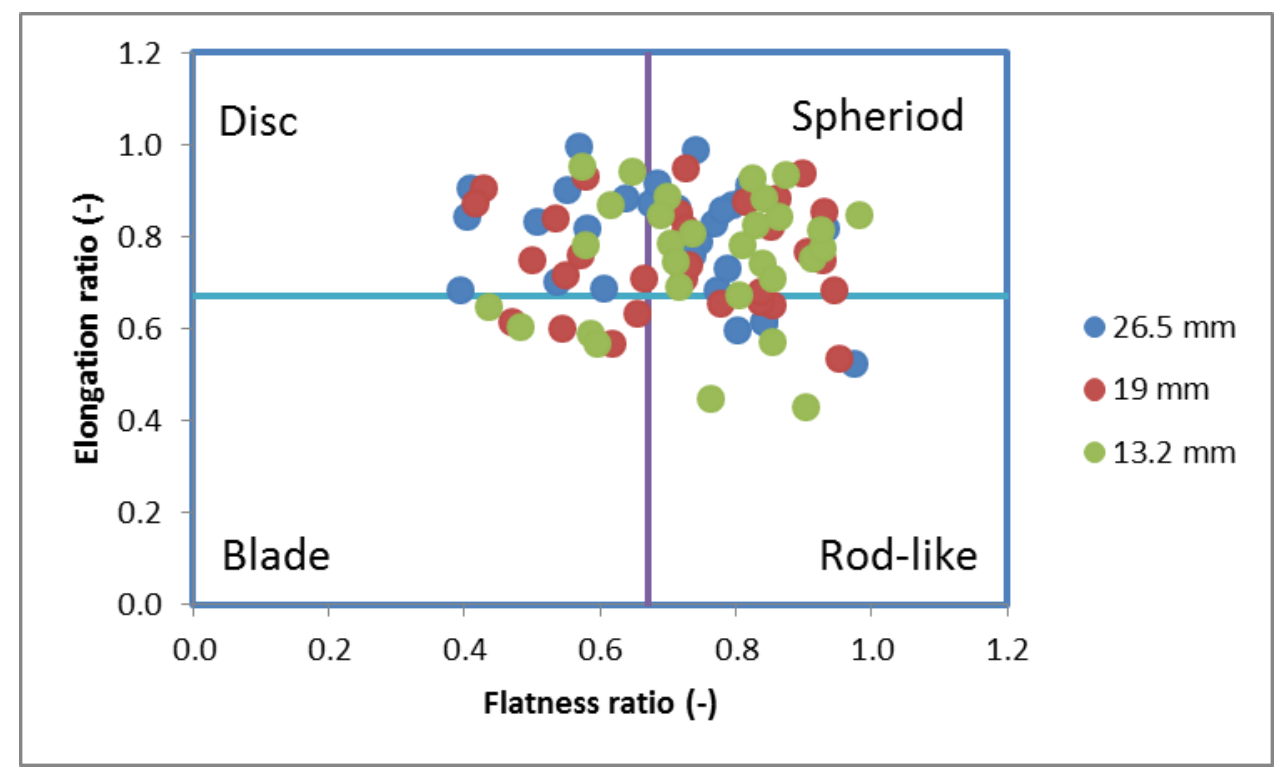

Figure 2. Classification of particle shape: Zingg diagram

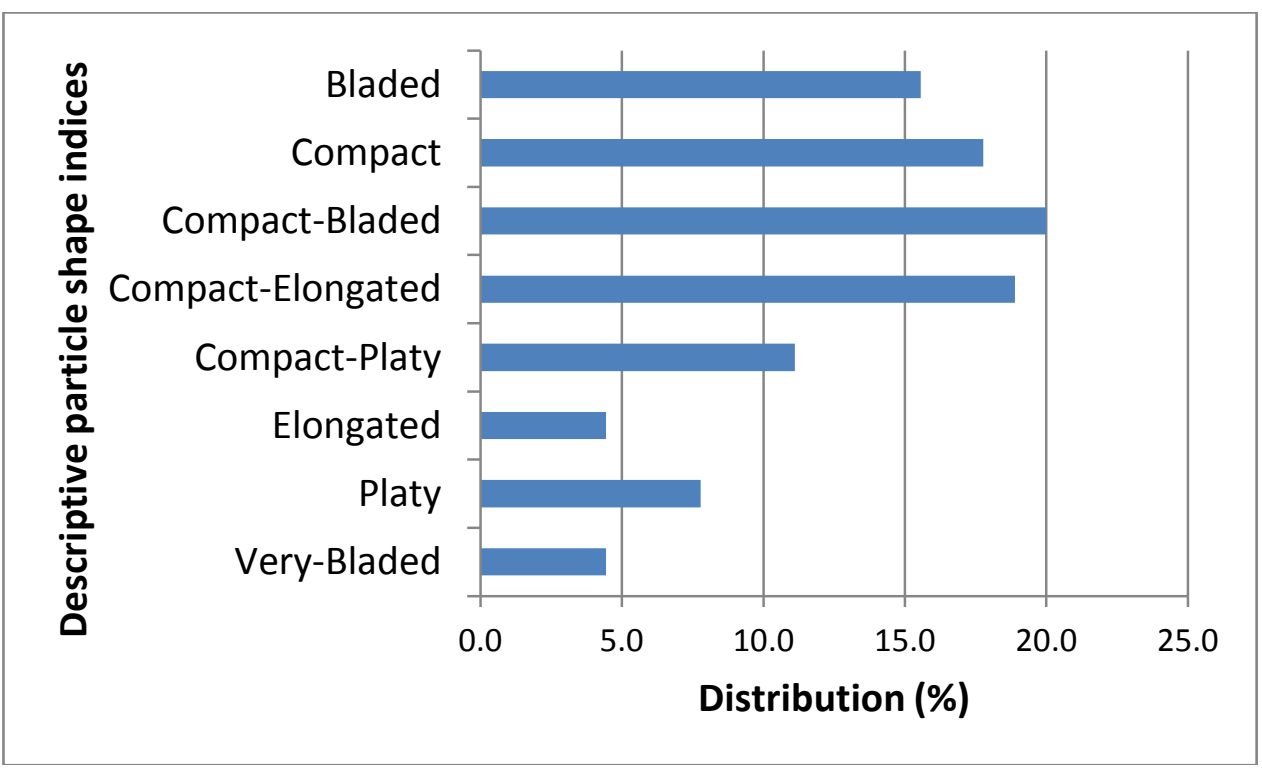

Figure 3. Classification of particle shape based on the triangulation diagram 


\section{Correlation between 3- $\mathrm{D}_{\text {sphericity }}$ and Aspect Ratio}

The results in figure 4 show that the association between $3-\mathrm{D}_{\text {sphericity }}\left(3-\mathrm{D}_{\mathrm{SPH}}\right)$ and aspect ratio (AR) is statistically significant with a non-linear equation. The non-linear equation describes $86 \%$ of the variability of $3-\mathrm{D}_{\mathrm{SPH}}$ with $\mathrm{AR}$ and shows a decrease in three dimensional sphericity with increasing aspect ratio.

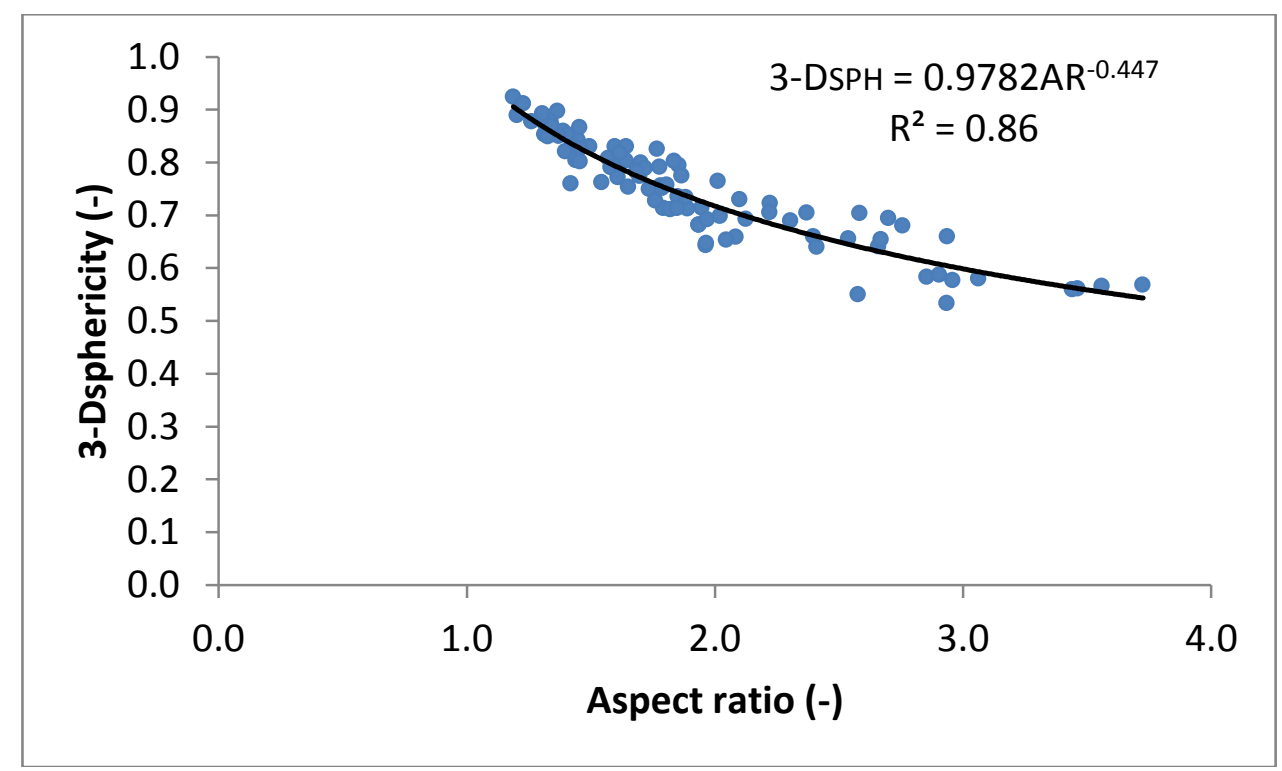

Figure 4. Variation of 3-Dsphericity with aspect ratio

\section{Correlation between Specific Surface Area and Particle Diameter}

The relationship between specific surface area (SSA) and the particle diameter $\left(\mathrm{d}_{\mathrm{p}}\right)$, defined by Equation 3 is presented in Figure 5. The results show that the relationship to describe the correlation between the two is non-linear. The equation is statistically significant describing $91 \%$ of the variability of SSA with particle diameter.

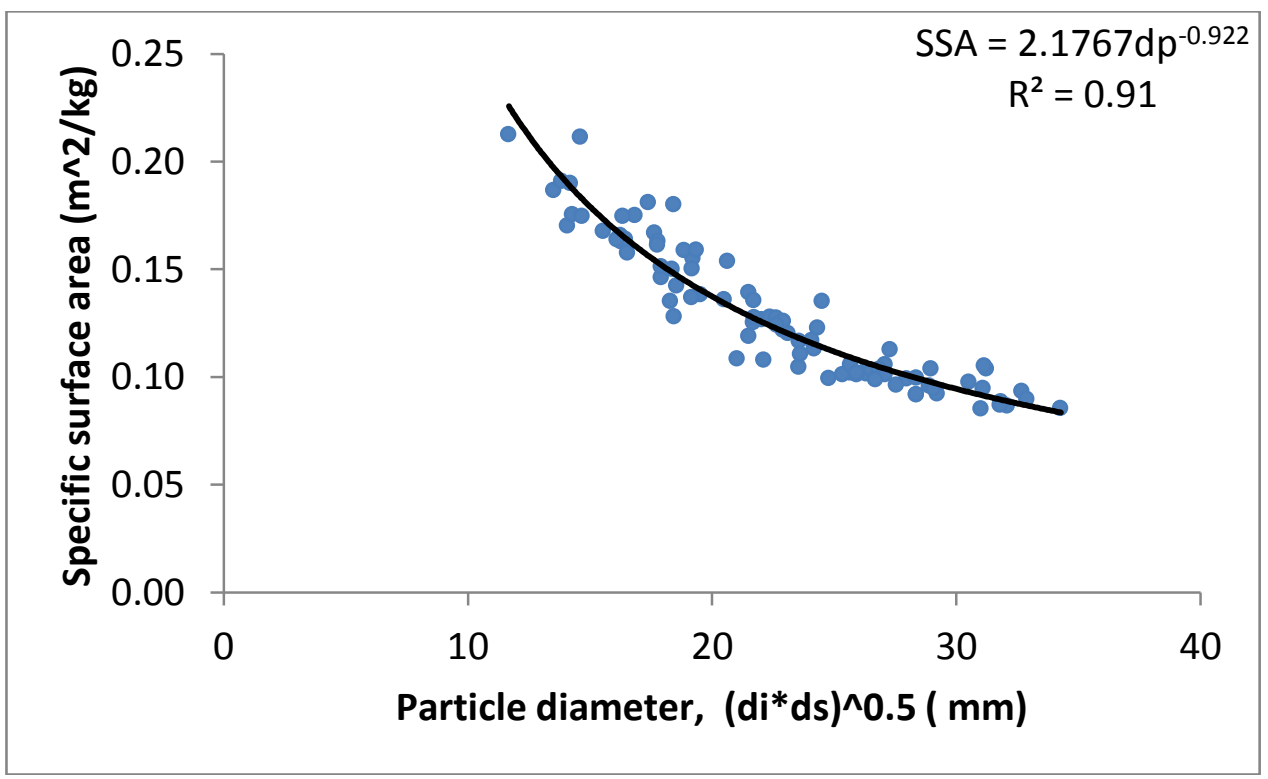

Figure 5. Variation of specific surface area with particle diamter (dp) 


\section{Distribution of Aspect Ratio and Form}

To examine how the aspect ratio (AR) and form were distributed with respect to the volume particle sizes, the cumulative probability distributions were computed and plotted together (Garboczi et. al., 2012). Figure 6 shows that the distribution of AR cannot be clearly differentiated with respect to particle size classes, $26.5 \mathrm{~mm}, 19.0 \mathrm{~mm}$ and $13.2 \mathrm{~mm}$. AR does not provide a consistent and significant difference between the different class sizes. On the other hand, while not significant, form as shown in Figure 7 shows a measure of size dependence. There is a noticeable difference between the class sizes that can be observed. This is more evident between class sizes $13.2 \mathrm{~mm}$ and $26.5 \mathrm{~mm}$ as well as between class sizes $19 \mathrm{~mm}$ and $26.5 \mathrm{~mm}$. For the same value for form, a different cumulative probability value is obtained for the different particle size class.

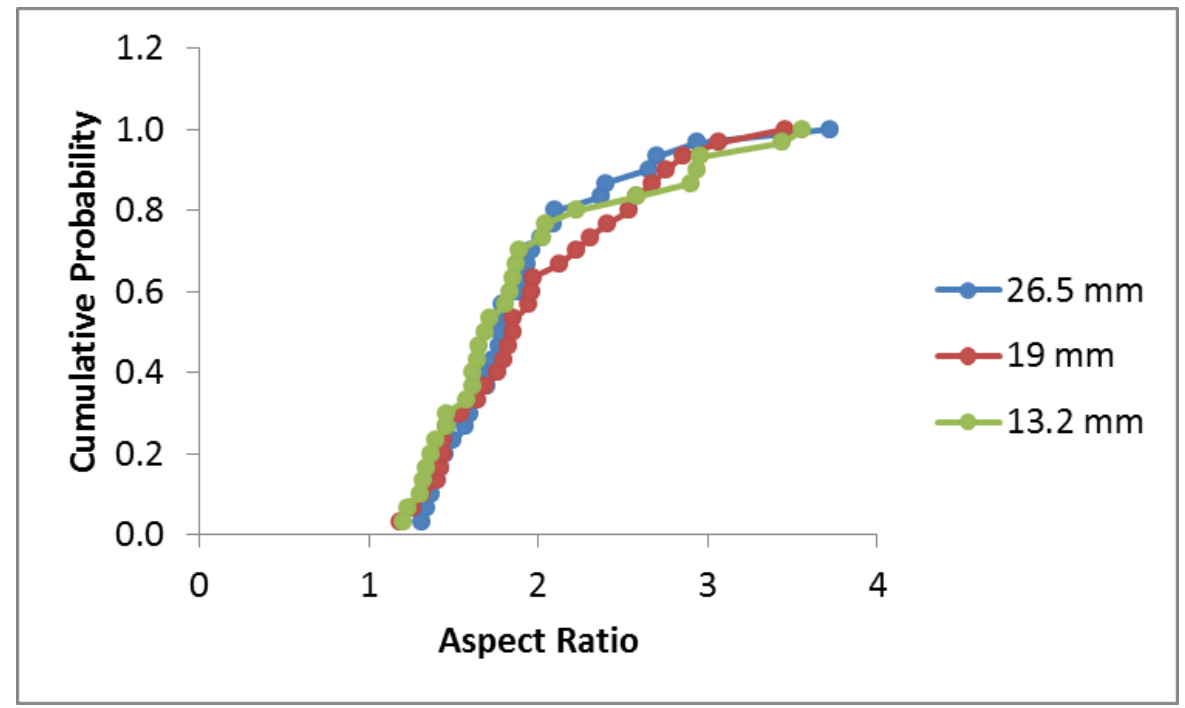

Figure 6. Cumulative probability for aspect ratio (AR)

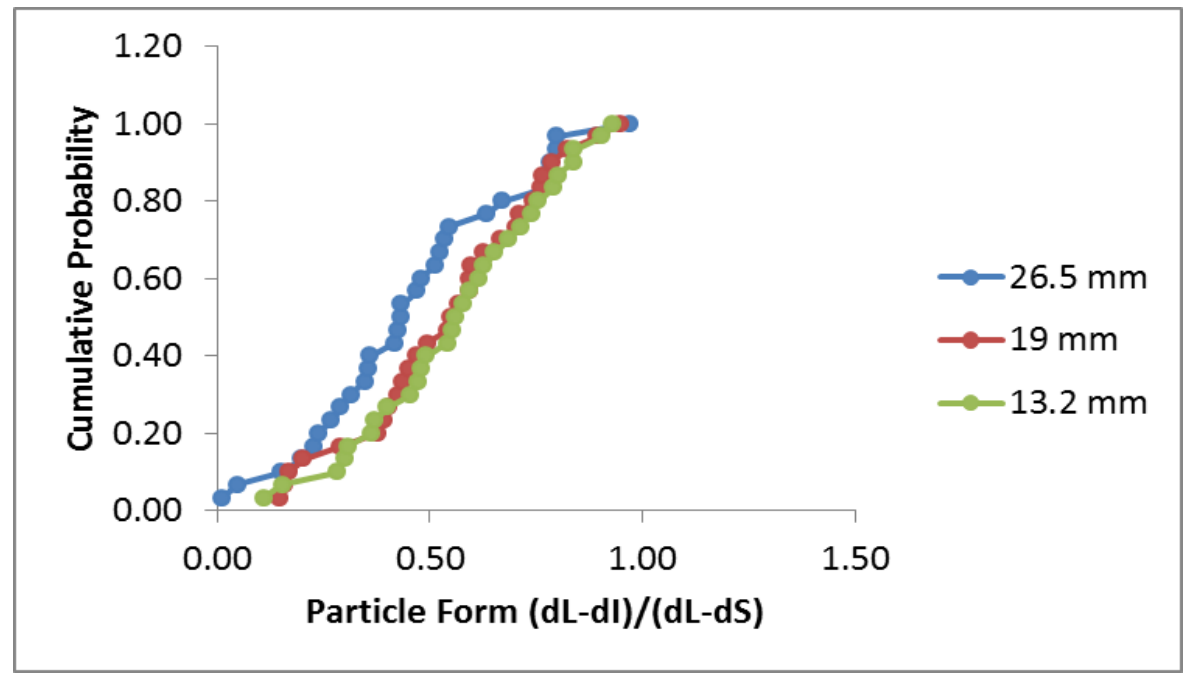

Figure 7. Cumulative probability for form 


\section{Distribution of 3-D sphericity and Specific Surface Area}

The distribution of 3-D $\mathrm{D}_{\mathrm{SPH}}$ with respect to the particle class sizes is shown in Figure 8. It shows mixed results, but close similarity exists. It can be stated that 3 - $\mathrm{D}_{\text {sphericity }}$ does not significantly distinguish between the class sizes. On the other hand SSA significantly discriminates between the class sizes, showing that SSA is dependent on particle size as shown in Figure. 9.

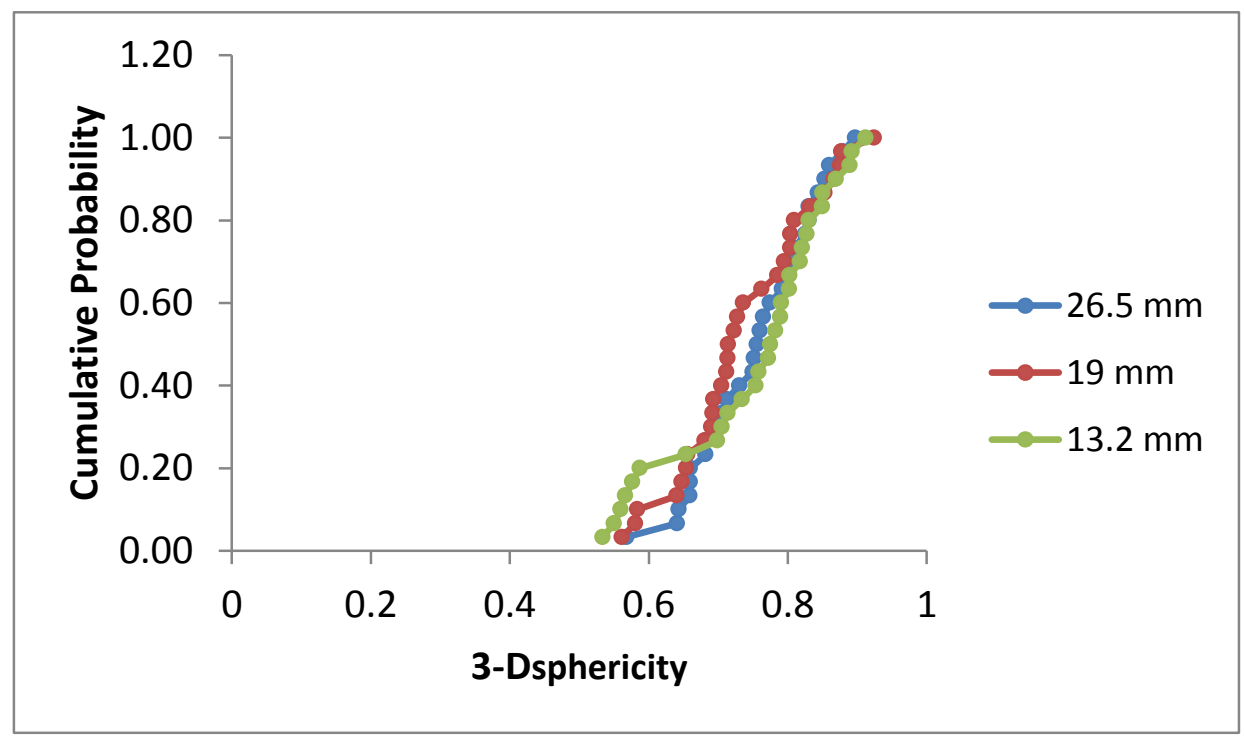

Figure 8. Cumulative probability for 3-Dsphericity

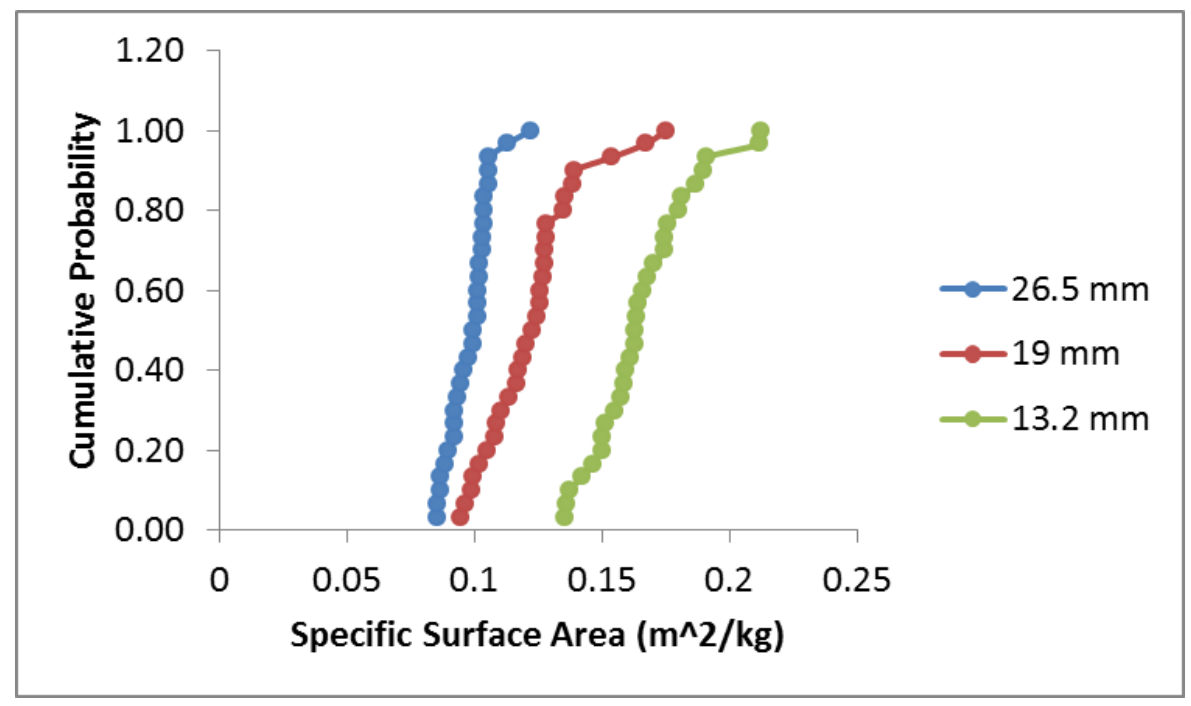

Figure 9. Cumulative probability for specific surface area (SSA)

\section{Distribution of Surface Area Ratio and SEVA}

From Figure 10 and Figure 11, it is clear that surface area ratio (SAR) and SEVA do not discriminate between the class sizes and are therefore independent of particle size. The observed results are expected since SAR and SEVA while defined differently are primarily formulated using the same values of volume and surface area of the particles. 


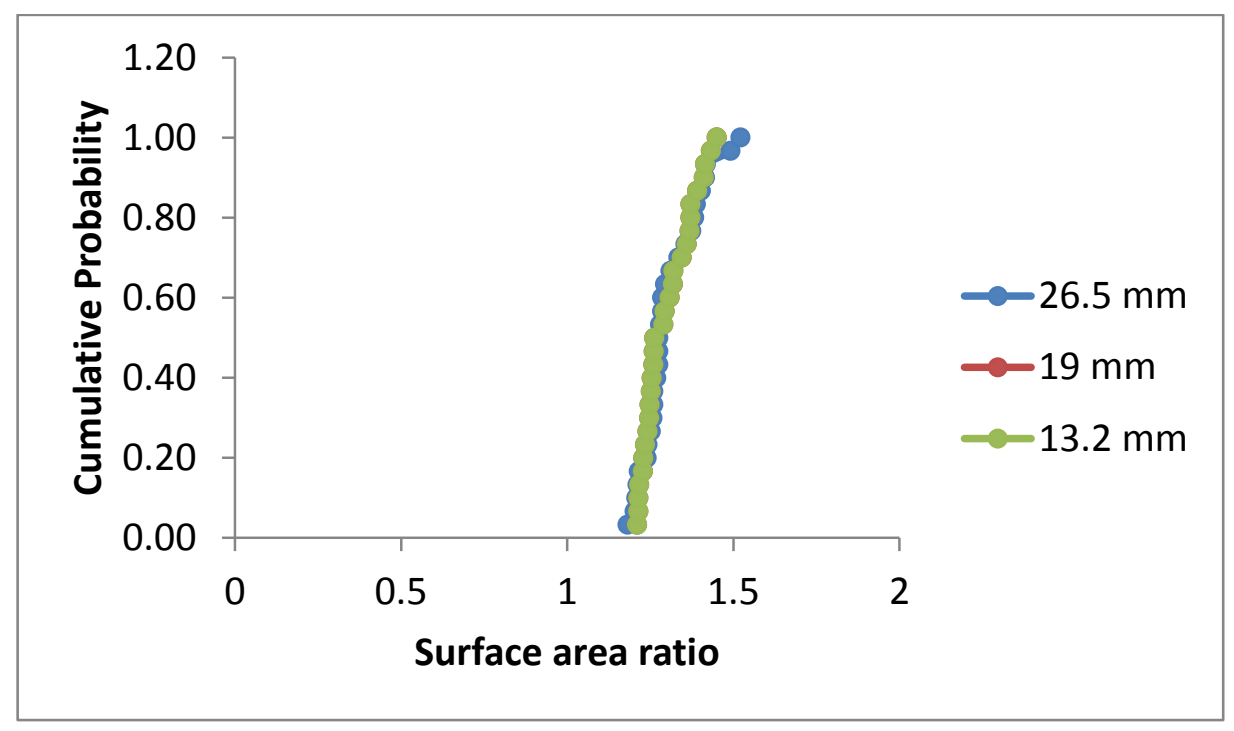

Figure 10. Cumulative probability for surface area ratio (SAR)

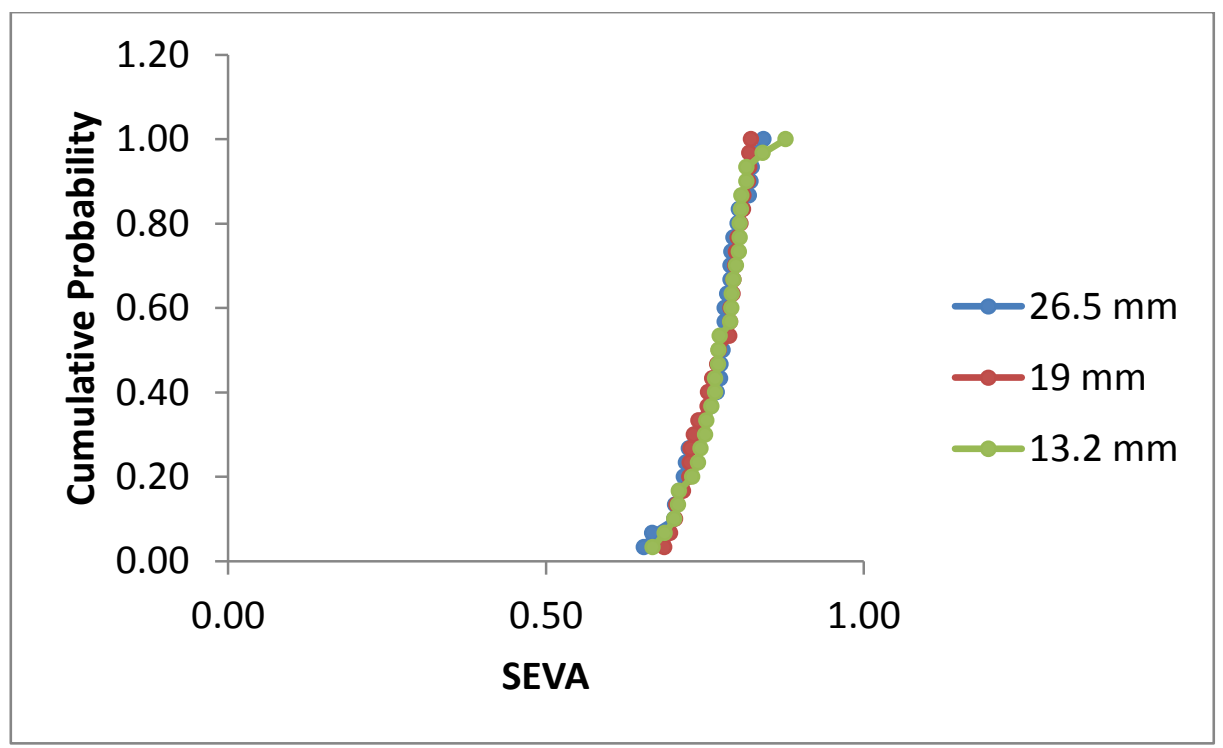

Figure 11. Cumulative probability for SEVA

The results show shape similarity or close similarity in terms of AR, 3-D $\mathrm{D}_{\mathrm{SPH}}$, SAR and SEVA, meaning that these parameters are independent of particle size. The results are consistent with the findings of Garboczi et. al., (2012), who used X-ray CT in their study. Form particle and SSA as presented in Figure 7 and Figure 9 respectively, capture the influence of the different scales of the particle size of the same sample.

\section{Conclusion}

The paper has presented some results of an on-going study on the characterisation of shape properties of aggregates using the 3-D laser scanner technology. The results of this study have shown that meaningful correlations exist between 3-D sphericity and aspect ratio as well as between specific surface area and the particle diameter as 
defined in this paper. The findings of the current study are consistent with generally accepted relationships between shape parameters and particle size, in that most shape parameters were found to be independent of particle size, thus validating the application of the 3-D laser scanning technology. Where it is necessary to quantify influence of particle size distribution on the bulk properties of pavement materials, the results presented have shown that specific surface area and particle Form can be used to discriminate between particle sizes of the same sample. The work presented is the first step towards establishing correlations, between the 3-D shape parameters, size of different types of aggregates from different sources and their influence on the bulk properties of pavement materials. The long term objective of the study is to produce knowledge and results which can be practically applied in the improvement of methods for pavement material characterisation that are based on aggregate shape characteristics. In addition, the aggregate shape characteristics are to be linked to pavement material performance.

\section{Acknowledgement}

Funding for 3-D laser scanning research project was provided by the CSIR Strategic Research Panel (SRP) project TA-2011-001. The authors would like to acknowledge this support.

\section{References}

Anochie-Boateng J. K, Komba, J J and Mvelase, G. M. (2013). "Three-dimensional laser scanning technique to quantify aggregate and ballast shape properties". Construction and Building Materials: 43: pp 389-398.

Anochie-Boateng JK, Komba J, Tutumluer E. (2012). "Aggregate surface areas quantified through laser measurements for South African asphalt mixtures". Journal of Transportation Engineering (ASCE);138(8): pp 1-11.

Prowell, B.D. Zhang, J and Brown, E.R,. (2005). "Aggregate Properties and the Performance of Superpave- Designed Hot Mix Asphalt”. NCHRP Report 539.

Das, A. (2006). “A revisit to Aggregate Shape Parameters". Workshop on Aggregates - Flakiness and Elongation Indices - (WSOA), New Delhi.

Garboczi E.J,. Liu, X , and Taylor, M.A (2012). "The 3-D shape of blasted and crushed rocks: From $20 \mu \mathrm{m}$ to 38mm". Powder Technology: 229, pp 84-89

Graham D.J, Midgley N.G (2000). "Graphical representation of particle shape using triangular diagrams: an Excel spreadsheet method". Earth Surface Processes and Landforms: 25(13): pp 1473-1477

Janoo, V. (1998). "Quantification of Shape, Angularity, and Surface Texture of Base Course Materials”. Special Report 98-1, US Army Corps of Engineers, Cold Regions Research \& Engineering Laboratory.

Lees, G. (1964). "The measurement of particle shape and its influence in engineering materials". Journal of the British Granite and Whinestone Federation, London, 4(2): pp 1-22. 
Pan T, Tutumluer E. (2010). "Imaging-based direct measurement of aggregate surface area and its application in asphalt mixture design". International Journal of Pavement Engineering: 11(5): pp 415-28.

Santamarina, J.C., and Cho, G.C. (2004). "Soil Behaviour: The Role of Particle Shape". In Proceedings of the Skempton Conference, London. 